\title{
Influence of Thickness on the Holographic Parameters of H-PDLC Materials
}

\author{
S. Gallego, ${ }^{1,2}$ M. Ortuño, ${ }^{1,2}$ A. Márquez, ${ }^{1,2}$ R. Fernández, ${ }^{1}$ M. L. Álvarez, ${ }^{1,2}$ \\ A. Beléndez, ${ }^{1,2}$ and I. Pascual ${ }^{1,3}$ \\ ${ }^{1}$ Instituto Universitario de Física Aplicada a las Ciencias y las Tecnologías, Universidad de Alicante, \\ Apartado 99, 03080 Alicante, Spain \\ ${ }^{2}$ Departamento de Física, Ingeniería de Sistemas y Teoría de la Señal, Universidad de Alicante, Apartado 99, 03080 Alicante, Spain \\ ${ }^{3}$ Departamento de Óptica, Farmacología y Anatomía, Universidad de Alicante, Apartado 99, 03080 Alicante, Spain \\ Correspondence should be addressed to S. Gallego; sergi.gallego@ua.es
}

Received 15 July 2014; Accepted 5 November 2014; Published 15 December 2014

Academic Editor: Michael R. Gleeson

Copyright (c) 2014 S. Gallego et al. This is an open access article distributed under the Creative Commons Attribution License, which permits unrestricted use, distribution, and reproduction in any medium, provided the original work is properly cited.

\begin{abstract}
For photopolymers the compound concentrations and final thickness of the sample should be known in order to model hologram formation and introduce the reaction-diffusion kinetics of the monomer-polymer system. In principle the cell thickness can be controlled by bead spacers between the two pieces of ITO glass. In this paper we report a study of the influence of thickness on the holographic properties of this type of materials. To fit the physical and optical thickness of the samples we used the rigorous coupled wave analysis assuming an exponential decay in the refractive index modulation.
\end{abstract}

\section{Introduction}

Photopolymers are one of the most promising holographic recording media for many technological applications, from integrated optical waveguide fabrication to optical data storage [1-5]. Their versatility is well known and new possibilities were created by including new components, such as nanoparticles or dispersed liquid crystal molecules in classical formulations, making them interesting for additional applications [6-8]. For example, the ability to control the diffraction efficiency of holographic optical elements by applying an electric field leads to the possibility of using HOEs in dynamic applications for agile beam steering, nonlinear optics, and optical switching devices [9].

Holographic polymer dispersed liquid crystals are known as H-PDLCs. They are made by holographic recording in a photopolymerization induced phase separation process (PIPS) in which the liquid crystal molecules diffuse to dark zones in the diffraction grating where they can be oriented by means of an electric field. The orientation of the liquid crystal produces a refractive index variation which changes the diffraction efficiency. Therefore, the grating develops a dynamic behavior that may be modified by electronic means. In this manner, it is possible to make dynamic devices such as tunable-focus lenses, sensors, phase modulators, or prism gratings [10-16].

There are many parameters involved in hologram formation. This process comprises a number of mechanisms, including photoinitiation, polymerization, diffusion, LC phase separation, and shrinkage. Several models have been developed for the formation of holograms in photopolymers [17-25]. The most recent models incorporate an initiation process, the peculiar properties of phase-separated LCs, development of anisotropic gratings, size of the polymer chains and scattering, and so forth [26]. As opposed to "dry" photopolymers where the quantity of solvent used up during the drying process must be measured in order to calculate the final component concentrations [27], in HPDLCs, it can be assumed in principle that the concentration of each component remains constant once it is deposited between two glass plates. These parameters are important in order to simulate the material behavior correctly during recording. Alternatively, using experimental performance optimization other parameters have been analyzed [28]. In this sense many researchers have explored different material recipes to achieve high diffraction efficiency [28], fast response time, 
low drive voltage, and so forth. Some of the effects analyzed are exposure intensity, exposure temperature, monomer functionality, LC concentration, NVP concentration, driving voltage, surfactant, fluorination, index matching, and nanoparticle doping [29]. Nevertheless, the effect of the thickness of the sample on grating morphology has not yet been studied in depth.

Usually the prepolymer syrup is prepared under dark conditions (or visible light at a wavelength where the dye does not absorb) and then sandwiched between two conductive ITO glass plates separated using glass microspheres. In order to obtain samples of the same physical thickness, a weight of $1 \mathrm{~kg}$ was placed over the layer for 5 minutes. As a result we obtained a probabilistic physical thickness distribution with values higher than the diameter of the microspheres used between the ITO glasses. Usually the HPDLC plates are thinner than $40 \mu \mathrm{m}$ in order to achieve the reorientation of the liquid crystal molecules applying an electric field not so big. The goal of this paper is to analyze the effect of this thickness variation on the holographic behavior of some chemical compositions of HPDLC materials. In particular the final thickness of the samples is directly related to the dye concentration required to obtain enough light absorption and energetic sensitivity without large attenuation in depth of the refractive index profile [30]. In this sense we analyzed the effect of dye concentration on the properties of the transmission diffraction gratings recorded. In order to achieve a deeper insight into this aspect, we analyzed the evolution of diffraction efficiency as a function of exposure and used the fitting of the angular response of the hologram around the first Bragg angle to calculate the physical and optical thickness [30] and attenuation of refractive index modulation in depth.

We used an algorithm based on the rigorous coupled wave theory [31] where an exponential decay in the refractive index modulation, $\Delta n$, is assumed [32] and $\alpha_{n}$ is the coefficient of the depth attenuation refractive index modulation. In order to apply the RCW to obtain the efficiency of the different orders we studied a periodic structure: the hologram was divided into $G$ different subgratings of thickness $d_{g}$ each. The total thickness of the hologram, $d$, can be obtained from the sum of the thicknesses of the different subgratings:

$$
d=\sum_{g=1}^{G} d_{g}
$$

where $G$ is the number of gratings retained.

Each subgrating is assumed to have a periodic dielectric permittivity of the form

$$
\varepsilon_{g}(x)=\sum_{h} \varepsilon_{g, h} \exp [j h K x],
$$

where $K$ is the modulus of the grating vector and $\varepsilon_{g, h}$ is the $h$ th Fourier component of the relative permittivity in the grating sublayer $g$, which can be expressed as

$$
\varepsilon_{g, h}=\varepsilon_{0, h} \exp \left[-\alpha_{n} \sum_{g^{\prime}=1}^{g} d_{g^{\prime}}\right] .
$$

It is important to use for H-PDLC gratings a rigorous wave analysis for many reasons. One of them is the proximity of the border between thick and thin holograms because of the small values of thickness analyzed between 13 and $37 \mu \mathrm{m}$ and the high values of the refractive index modulation stored between 0.005 and 0.036 . In addition it is important to note that along this work we mention the index modulation, $\Delta n$, which is the index modulation at the beginning of the grating, since the depth attenuation on the last part of the material is clearly smaller. Finally we want to remark that for the reading beam used in this work our material is $100 \%$ transparent; nevertheless part of the energy is reflected due to the Fresnel losses for TE polarization; in addition due to the scattering the final DE measured will be reduced. This scattered energy depends on the coefficient of attenuation and scattering, $\alpha_{s}$, as follows:

$$
\mathrm{DE}=e^{-\alpha_{s} d / \cos \theta} \mathrm{DE}
$$

where $d$ is the physical thickness of the layer and $\theta$ is the angle of the beam with respect to the normal inside the hologram.

\section{Experimental Setup}

The monomer used was dipentaerythritol penta-/hexaacrylate (DPHPA) with a refractive index $n=1.490$. We used the nematic liquid crystal, Licristal BL036 from Merck. It is a mixture of 4-cyanobiphenyls with alkyl chains of different lengths. It has an ordinary refractive index $n_{0}=1.5270$ and a difference between extraordinary and ordinary index $\Delta n=0.2670$ [28]. There is a difference of 0.037 between the ordinary refractive index of the liquid crystal and that of the monomer.

The liquid crystal concentration was set at $28 \mathrm{wt} \%$ as the starting point for component optimization and remained practically unchanged during this process.

$\mathrm{N}$-vinyl-2-pyrrolidone was used as crosslinker, N-phenylglicine (NPG) as radical generator, octanoic acid (OA) as cosolvent [28], and ethyl eosin (YEt) as dye. N-methyl-2pyrrolidone was used in combination with NVP in order to control overmodulation during hologram recording.

The prepolymer solution was made by mixing the components under red light where the material is not sensitive. The solution was sonicated in an ultrasonic bath, deposited between two conductive ITO glass plates $1 \mathrm{~mm}$ thick and separated using two types of glass microspheres. The first type of microspheres was provided by Aldrich with a thickness between 9 and $13 \mu \mathrm{m}$ and the second type was provided by Whitehouse Scientific with a thickness between 20 and $30 \mu \mathrm{m}$. The device was exposed to a laser beam $(\lambda=532 \mathrm{~nm})$ in a holographic setup in order to record a diffraction grating in the photopolymer layer. A photopolymerization reaction takes place in the bright zones of the diffraction grating and a highly reticulated polymer network is generated. The liquid crystal molecules diffuse to the unexposed region where they remain as droplets. In this work we used three different chemical compositions and the refractive indexes of the final mixture are represented in Table 1 . In the first two chemical compositions we analyzed the influence of 
TABLE 1: Composition of photopolymers 1,2, and 3 in wt $\%$ and the refractive index of the final mixture.

\begin{tabular}{lcccccccc}
\hline Photopolymer & DPHPA & BL036 & YEt & NPG & NVP & OA & NMP & $n$ \\
\hline 1 & 48.4 & 29.2 & 0.1 & 1.5 & 16.4 & 4.4 & 0.0 & 1.535 \\
2 & 48.4 & 29.2 & 0.2 & 1.5 & 16.3 & 4.4 & 0.0 & 1.535 \\
3 & 46.9 & 28.3 & 0.1 & 1.5 & 15.8 & 4.2 & 3.1 & 1.532 \\
\hline
\end{tabular}

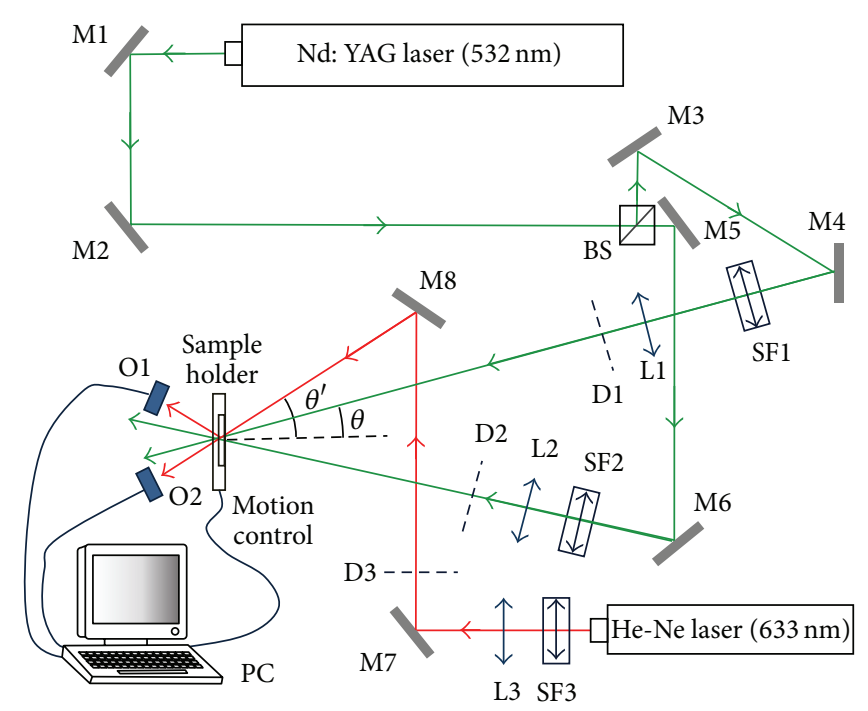

FIGURE 1: Experimental setup. BS: beamsplitter, Mi: mirror, SFi: spatial filter, Li: lens, Di: diaphragm, Oi: optical power meter, and PC: data recorder.

the dye concentration on the optical thickness of the diffraction gratings. In the case of composition 3, the analysis described in [28] was done to control overmodulation and obtain stable maximum diffraction efficiency.

We monitored the diffraction grating in real time using red light $(\lambda=633 \mathrm{~nm})$ where the dye does not absorb. After recording, when the green laser was shut off, we rotated the sample to record the angular response around the Bragg angle and obtained the data necessary to calculate the physical and optical thickness of the holographic diffraction gratings.

The experimental device is shown in Figure 1. An Nd:YAG laser tuned at a wavelength of $532 \mathrm{~nm}$ was used to record diffraction gratings by means of continuous laser exposure. The laser beam was split into two secondary beams with an intensity ratio of $1: 1$. The diameter of these beams was increased to $1 \mathrm{~cm}$ using a spatial filter and collimating lens, while spatial filtering was ensured. The object and reference beams were recombined at the sample at an angle of 16 degrees to the normal with an appropriate set of mirrors, and the spatial frequency obtained was 1036 lines $/ \mathrm{mm}$. The working intensity at $532 \mathrm{~nm}$ was $7 \mathrm{~mW} / \mathrm{cm}^{2}$, and the exposure time was $60 \mathrm{~s}$. The diffracted and transmitted intensity were monitored in real time with a He-Ne laser positioned at Bragg's angle (19.1 $1^{\circ}$ tuned to $633 \mathrm{~nm}$, where the material does not polymerize. The diffraction efficiency was calculated as the ratio of the diffracted beam $\left(I_{D}\right)$ to the incident power $\left(I_{0}\right)$.

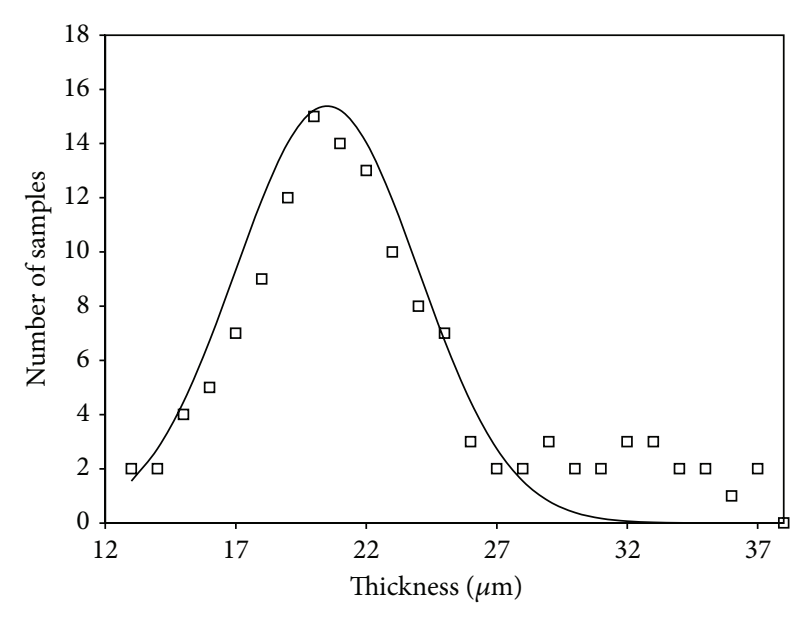

FIGURE 2: Number of samples as a function of the physical thickness using chemical composition 1 and microspheres $13 \mu \mathrm{m}$ in diameter, hollow squares. Gaussian distribution, solid line.

\section{Results and Discussion}

In this section we present the results obtained after recording hundreds of diffraction gratings between 13 and $40 \mu \mathrm{m}$. As we explained in the introduction, the physical thicknesses of the samples are always greater than the diameter of the microspheres. The values obtained using the $13 \mu \mathrm{m}$ thick Aldrich microspheres and chemical composition 1 for 135 samples are shown in Figure 2. We can estimate the error in calculating the thickness, using the method described in the introduction section, at less than $1 \mu \mathrm{m}$. We can see that a high probability occurs between 20 and $21 \mu \mathrm{m}$. In addition $70 \%$ of the samples have a thickness between 17 and $25 \mu \mathrm{m}$. Comparing these results, with a Gaussian distribution with a mean or expectation equal to 20.5 and standard deviation equal to 3.5 , to the experimental data, we can verify a similar behavior. Figure 3 shows one angular response fitted using the algorithm based on RCW taking into account the exponential decay of the refractive index modulation. In particular Figure 3 corresponds to the $29 \mu \mathrm{m}$ thick grating with a refractive index modulation of 0.015 , an attenuation coefficient for the refractive index modulation, $\alpha_{n}$, of $0.03 \mu \mathrm{m}^{-1}$, and a coefficient of attenuation and scattering, $\alpha_{s}$, of $0.005 \mu \mathrm{m}^{-1}$. Analyzing this figure the clear effects of the overmodulation of the refractive index can be detected. This figure shows good agreement between the experimental data and the simulation provided by the algorithm [32] as well as a smoothing of the secondary lobes due to the attenuation in depth of the refractive index that justify the use of 


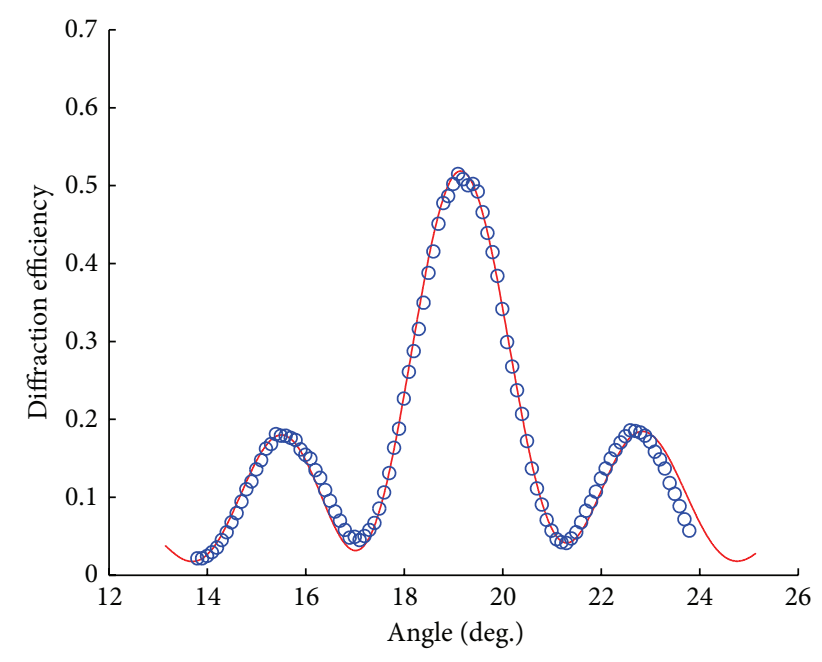

FIGURE 3: Diffraction efficiency as a function of the readout angle for a grating $29 \mu \mathrm{m}$ thick, hollow circles, and the fitting provided by the algorithm using composition 1.

the algorithm to fit the angular scan and measure the physical and optical thickness.

3.1. Effect of Dye Concentration. Ethyl eosin, the dye, is bleached like the typical xanthene dyes during the photoinitiation process [28]. Bearing this in mind, a high dye concentration is required for applications like multiplexing data pages, where the thickness of the samples is greater than $250 \mu \mathrm{m}$. However, a high concentration of dye increases scattering and damages the morphology of the recorded holograms [33]. Furthermore, increasing the dye concentration can modify the optical thickness of the diffraction grating or the attenuation of the refractive index modulation in depth [30].

As opposed to photopolymer holographic memories, $\mathrm{H}$ PDLC materials contain mainly polyfunctional monomers in order to achieve an effective PIPS effect. Polyfunctional monomers give rise to a high degree of reticulation of the polymer chains in a short time and therefore high refractive index modulation at a lower exposure than do standard photopolymers without liquid crystals. For this reason, the dye content in H-PDLC materials, where very thin layers are required, must be very small. In the literature the concentration of the dye YEt changes in the range of $0-$ $0.7 \%$ normally used when a dye is optimized in standard photopolymers [28]. In this work we used two compositions, 1 and 2, that had given us good results in previous studies. In order to avoid an undesired increase in scattering with composition 2, we used the microspheres provided by Whitehouse Scientific to increase the physical thickness slightly. The diffraction efficiency during 400 s of exposure for different ranges of thicknesses is shown in Figure 4: Figure 4(a) for composition 1 and Figure 4(b) for composition 2. As can be seen for composition 1 the maximum DE is achieved with an exposure of around $90 \mathrm{~mJ} / \mathrm{cm}^{2}$ and for composition 2 of around $70 \mathrm{~mJ} / \mathrm{cm}^{2}$. Taking into account results for not coverplated photopolymers [34], where an increase in thickness produces an important increase in the energetic sensitivity of the material, important changes can be expected in the holographic behavior of the HPDLC layers. However, from an initial analysis of the two figures it is hard to clarify the specific influence of thickness on diffraction efficiency during recording. The diffraction behavior looks very similar. At best we can say that the gratings with low values of thickness exhibit slightly better energetic sensitivity. It is important to note that we defined this parameter as the exposure required to achieve the maximum value of $\mathrm{DE}$, or in other words how fast is the increase of the DE as a function of time after the inhibition period. In other words, they achieve maximum DE faster than the thicker ones. All the gratings have the same spatial frequency; therefore, we know that the diffraction efficiency depends on the product of optical thickness and refractive index modulation [35]. If these layers have different thicknesses, obviously they have different refractive index modulations.

In order to delve deeper into this phenomenon and obtain quantitative information about the refractive index modulation, we fitted the angular responses of different gratings just a few seconds after exposure. In Figure 5 we present the results for both, composition 1 and composition 2 , the inverse of the average refractive index modulation as a function of the thickness. The linearity in this behavior, for both cases, means that the product of thickness and refractive index modulation remains almost constant. These values were measured after $60 \mathrm{~s}$ of exposure, when the gratings are in the saturation zone and the polymerization has finished for a range of thickness between 13 and $37 \mu \mathrm{m}$. The results obtained for composition 2 are similar. We will compare directly the two dye concentrations at the end of this section using the data fitted from the angular response. The data presented in Figure 5 suggest that the concentration of components in the sample decreases with increasing thickness. We measure the area of the polymer layer for each sample and it is always around $4 \mathrm{~cm}^{2}$. Using the proposed algorithm to analyze the angular response around Bragg's angle we also obtain the values of $\alpha_{s}$ and $\alpha_{n}$. As can be expected the average of these values decreases with thickness. For composition 1 the thinnest layers present values of $\alpha_{n}$ up to $0.09 \mu \mathrm{m}^{-1}$ and of $\alpha_{s}$ up to $0.06 \mu \mathrm{m}^{-1}$, whereas the values of both these parameters for thicker layers (over $30 \mu \mathrm{m}$ ) are around $0.03 \mu \mathrm{m}^{-1}$.

To complete our study of the influence of the dye concentration, it is necessary to compare chemical compositions 1 and 2 using the same range of thicknesses. The results (average values) are presented in Table 2. From a comparison of the two chemical compositions it may be seen that if the dye concentration is doubled, the refractive index increases; however, there is also an undesirable increase in parameters such as $\alpha_{s}$ and $\alpha_{n}$. A high value of $\alpha_{s}$ implies a lower quality in the recorded hologram and the DE; on the other hand, $\alpha_{n}$ only affects the optical thickness and the angular selectivity around Bragg's angle; thus $\alpha_{n}$ is less critical than $\alpha_{s}$.

Let us present the direct comparison during recording for compositions 1 and 2. In Figure 6 we can see the obvious 


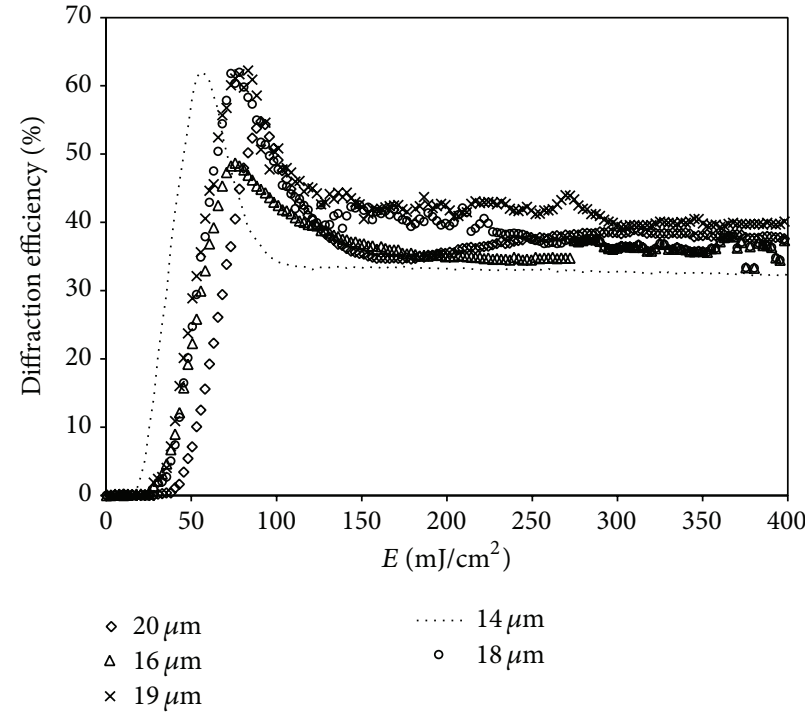

(a)

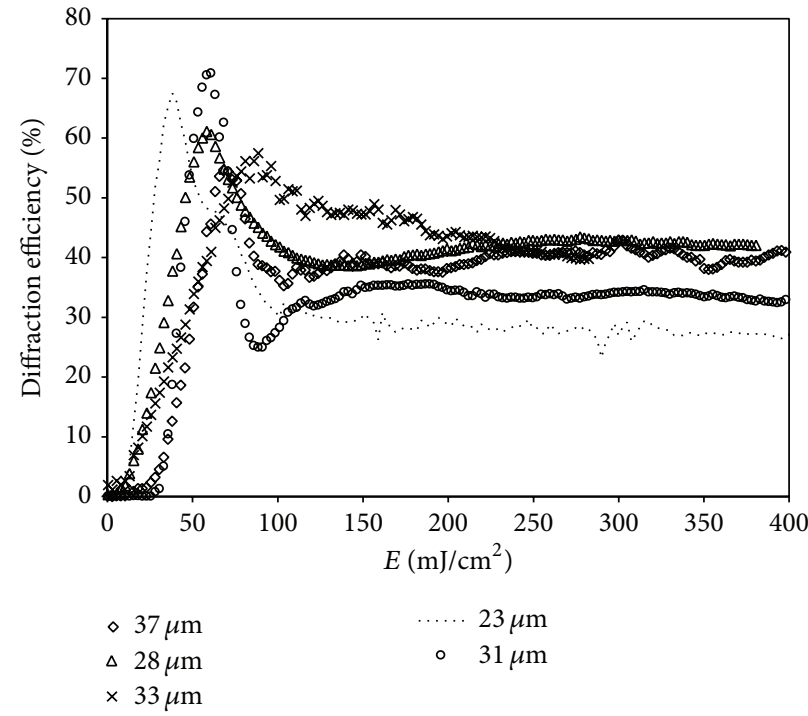

(b)

FIGURE 4: (a) Diffraction efficiency as a function of recording exposure; sample thickness between 14 and $20 \mu \mathrm{m}$ using composition 1 . (b) Diffraction efficiency as a function of recording exposure; sample thickness between 23 and $37 \mu \mathrm{m}$ using composition 2 .

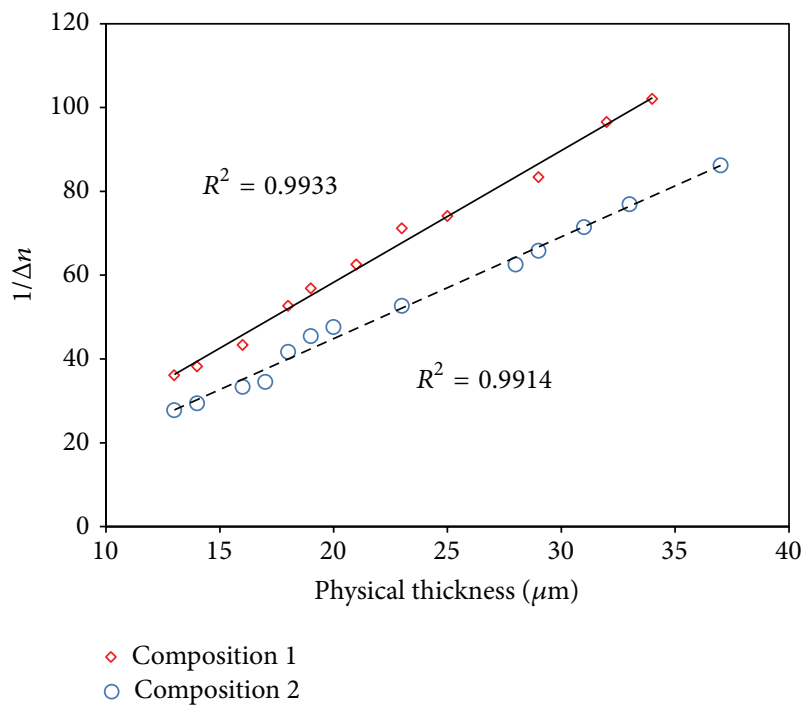

FIGURE 5: The inverse of the refractive index modulation as a function of physical thickness for the layers with chemical composition 1.

different behavior of these compounds under sinusoidal illumination; nevertheless the final DE is very similar: $50 \%$ for composition 1 and $40 \%$ for composition 2. After fitting of the angular response we can determine that both samples have the same physical thickness $(30 \pm 1) \mu \mathrm{m}$; however, the material with higher dye concentration presents better energetic sensitivity and higher value of refractive index modulation; the DE after $100 \mathrm{~s}$ of exposure is low due to the overmodulation effects. It is important to note that the maximum of DE is smaller, $77 \%$ versus $82 \%$ for composition 1 , due to the larger value of $\alpha_{s}$, scattering coefficient. In this
TABLE 2: Main parameters fitted from the angular response for chemical compositions 1 and 2 after recording.

\begin{tabular}{lcccc}
\hline $\begin{array}{l}\text { Chemical } \\
\text { composition }\end{array}$ & $\begin{array}{c}\text { Thickness } \\
(\mu \mathrm{m})\end{array}$ & $\begin{array}{c}\text { Refractive index } \\
\text { modulation }\end{array}$ & $\begin{array}{c}\alpha_{n} \\
\left(\mu \mathrm{m}^{-1}\right)\end{array}$ & $\begin{array}{c}\alpha_{s} \\
\left(\mu \mathrm{m}^{-1}\right)\end{array}$ \\
\hline 1 & 30 & 0.011 & 0.03 & 0.03 \\
2 & 30 & 0.014 & 0.05 & 0.04 \\
1 & 19 & 0.018 & 0.04 & 0.04 \\
2 & 19 & 0.023 & 0.06 & 0.06 \\
\hline
\end{tabular}

sense, it is important to remark that the presented data are not corrected to take into account the Fresnel losses. Taking into account the maximum DE, TE polarization is around $86 \%$.

3.2. Layers without Overmodulation. As we have shown in a previous paper, incorporation of 3\% NMP in the H-PDLC formulation leads to an improved result in order to obtain higher values of diffraction efficiency after saturation [28]. The diffraction efficiency curve is stabilized with a high value of $\mathrm{DE}$ preventing overmodulation during hologram recording. This makes it possible to obtain a high DE max at the end of the exposure and therefore a better performance in photopolymers with ethyl eosin as dye. In this section we present the same study for composition 3. Figure 7 shows diffraction efficiency as a function of exposure for four different thicknesses. In this case after saturation the diffraction efficiency remains close to the maximum due to the presence of NMP. As, in the case of Figure 4, the four different thicknesses present very similar behaviors and energetic sensitivity. We followed the same steps to calculate the data in Table 3 and from this table we can extract similar results. 


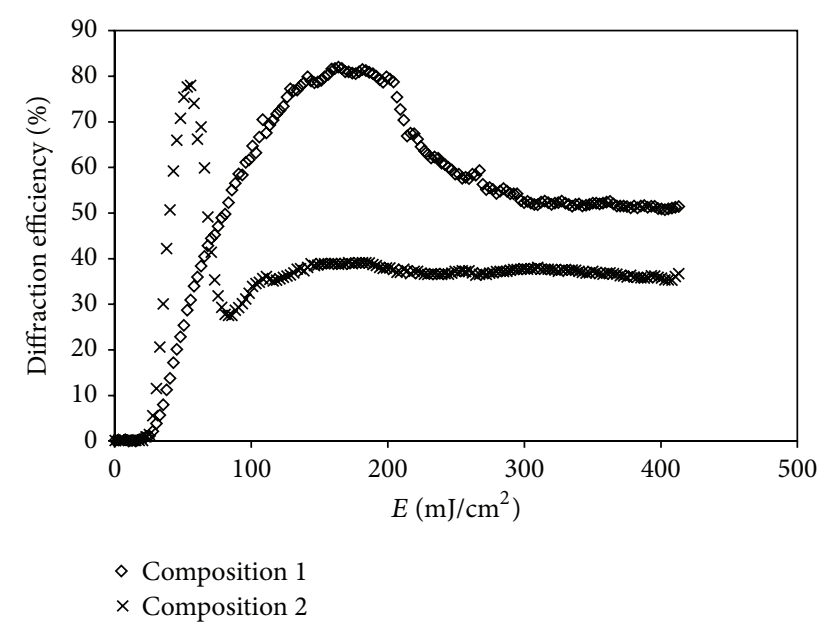

FIGURE 6: Comparison between the recording processes for compositions 1 and 2 for layers $30 \mu \mathrm{m}$ thick.

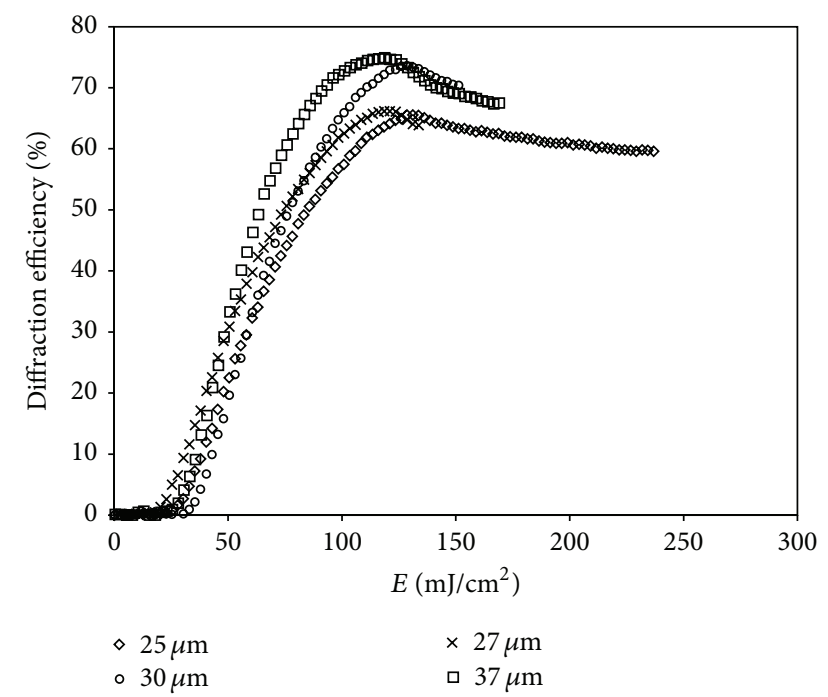

FIGURE 7: Diffraction efficiency as a function of recording exposure; sample thickness between 25 and $37 \mu \mathrm{m}$ using composition 3 .

An increase in thickness implies a low concentration of the component in the syrup due to the capillarity action of the prepolymer syrup and the associate adhesive forces between the liquid and ITO glass substrate; thus the refractive index modulation decreases more or less linearly, and parameters such as $\alpha_{s}$ and $\alpha_{n}$ decrease too.

\section{Conclusions}

We analyzed the effects of thickness on the final holographic properties of H-PDLC materials with different chemical compositions. We demonstrated that the product of refractive index modulation and thickness (diffraction efficiency) remains constant for layers with the same chemical composition over a wide range of thicknesses due to the inverse, linear variation of these two parameters. In addition we showed that an increase in dye concentration affects the depth attenuation
TABLE 3: Main parameters fitted from the angular response for chemical composition 3.

\begin{tabular}{lccc}
\hline $\begin{array}{l}\text { Thickness } \\
(\mu \mathrm{m})\end{array}$ & $\begin{array}{c}\text { Refractive index } \\
\text { modulation }\end{array}$ & $\alpha_{n}\left(\mu \mathrm{m}^{-1}\right)$ & $\alpha_{s}\left(\mu \mathrm{m}^{-1}\right)$ \\
\hline 25 & 0.0087 & 0.09 & 0.012 \\
27 & 0.0083 & 0.09 & 0.012 \\
31 & 0.0069 & 0.08 & 0.004 \\
37.5 & 0.0061 & 0.07 & 0.002 \\
\hline
\end{tabular}

of the hologram and scattering. Therefore, we underline the importance of controlling the thickness of H-PDLC materials in order to achieve high values of reproducibility in all the holographic parameters not only in diffraction efficiency.

\section{Conflict of Interests}

The authors declare that there is no conflict of interests regarding the publication of this paper.

\section{Acknowledgments}

The work was supported by the "Ministerio de Economía y Competitividad" of Spain under Projects FIS2011-29803C02-01 and FIS2011-29803-C02-02 and by the "Generalitat Valenciana" of Spain under Projects PROMETEO/2011/021 and ISIC/2012/013.

\section{References}

[1] J. Neumann, K. S. Wieking, and D. Kip, "Direct laser writing of surface reliefs in dry, self-developing photopolymer films," Applied Optics, vol. 38, no. 25, pp. 5418-5421, 1999.

[2] X. T. Li, A. Natansohn, and P. Rochon, "Photoinduced liquid crystal alignment based on a surface relief grating in an assembled cell," Applied Physics Letters, vol. 74, no. 25, pp. 3791-3793, 1999.

[3] A. Márquez, S. Gallego, M. Ortuño et al., "Generation of diffractive optical elements onto a photopolymer using a liquid crystal display," in Optical Modelling and Design, vol. 7717 of Proceedings of SPIE, May 2010.

[4] M. D. Lechner, "Photopolymers for optical memories and waveguides," in Electronic Properties of Polymers and Related Compounds, vol. 63 of Springer Series in Solid-State Sciences, pp. 301308, Springer, New York, NY, USA, 1985.

[5] H. J. Coufal, D. Psaltis, and G. T. Sincerbox, Eds., Holographic Data Storage, Springer, Berlin, Germany, 2000.

[6] N. Suzuki and Y. Tomita, "Silica-nanoparticle-dispersed methacrylate photopolymers with net diffraction efficiency near 100\%," Applied Optics, vol. 43, no. 10, pp. 2125-2129, 2004.

[7] E. Hata, K. Mitsube, K. Momose, and Y. Tomita, "Holographic nanoparticle-polymer composites based on step-growth thiolene photopolymerization," Optical Materials Express, vol. 1, no. 2, pp. 207-222, 2011.

[8] M. A. Ellabban, I. Drevenšek-Olenik, and R. A. Rupp, "Huge retardation of grating formation in holographic polymerdispersed liquid crystals," Applied Physics B: Lasers and Optics, vol. 91, no. 1, pp. 11-15, 2008. 
[9] M. S. Li, A. Y.-G. Fuh, J.-H. Liu, and S.-T. Wu, "Bichromatic optical switch of diffractive light from a BCT photonic crystal based on an azo component-doped HPDLC," Optics Express, vol. 20, no. 23, pp. 25545-25553, 2012.

[10] J. Yan, L. Rao, M. Jiao, Y. Li, H.-C. Cheng, and S.-T. Wu, "Polymer-stabilized optically isotropic liquid crystals for nextgeneration display and photonics applications," Journal of Materials Chemistry, vol. 21, no. 22, pp. 7870-7877, 2011.

[11] T. J. Bunning, L. V. Natarajan, V. P. Tondiglia, and R. L. Sutherland, "Holographic polymer-dispersed liquid crystals $(\mathrm{H}-$ PDLCs)," Annual Review of Materials Science, vol. 30, pp. 83-115, 2000.

[12] H. Ren, S. Xu, and S. T. Wu, "Gradient polymer network liquid crystal with a large refractive index change," Optics Express, vol. 20, no. 24, pp. 26464-26472, 2012.

[13] V. K. S. Hsiao, C. Lu, G. S. He et al., "High contrast switching of distributed-feedback lasing in dye-doped H-PDLC transmission grating structures," Optics Express, vol. 13, no. 10, pp. 37873794, 2005.

[14] S. Massenot, J.-L. Kaiser, M. C. Perez, R. Chevallier, and J.-L. de Bougrenet de La Tocnaye, "Multiplexed holographic transmission gratings recorded in holographic polymer-dispersed liquid crystals: static and dynamic studies," Applied Optics, vol. 44, no. 25, pp. 5273-5280, 2005.

[15] M. S. Li, S. T. Wu, and A. Y. G. Fuh, "Sensor for monitoring the vibration of a laser beam based on holographic polymer dispersed liquid crystal films," Optics Express, vol. 18, no. 25, pp. 26300-26306, 2010.

[16] M. Infusino, A. de Luca, V. Barna, R. Caputo, and C. Umeton, "Periodic and aperiodic liquid crystal-polymer composite structures realized via spatial light modulator direct holography," Optics Express, vol. 20, no. 21, pp. 23138-23143, 2012.

[17] G. Zhao and P. Mouroulis, "Diffusion model of hologram formation in dry photopolymers materials," Journal of Modern Optics, vol. 41, pp. 1929-1939, 1994.

[18] D. J. Lougnot, P. Jost, and L. Lavielle, "Polymers for holographic recording: VI. Some basic ideas for modelling the kinetics of the recording process," Pure and Applied Optics, vol. 6, no. 2, pp. 225-245, 1997.

[19] S. Piazzolla and B. K. Jenkins, "First-harmonic diffusion model for holographic grating formation in photopolymers," Journal of the Optical Society of America B: Optical Physics, vol. 17, no. 7, pp. 1147-1157, 2000.

[20] J. T. Sheridan and J. R. Lawrence, "Nonlocal-response diffusion model of holographic recording in photopolymer," Journal of the Optical Society of America A: Optics and Image Science, and Vision, vol. 17, no. 6, pp. 1108-1114, 2000.

[21] V. Moreau, Y. Renotte, and Y. Lion, "Characterization of DuPont photopolymer: determination of kinetic parameters in a diffusion model," Applied Optics, vol. 41, no. 17, pp. 3427-3435, 2002.

[22] C. Neipp, S. Gallego, M. Ortuño et al., "First-harmonic diffusion-based model applied to a polyvinyl-alcohol-acrylamidebased photopolymer," Journal of the Optical Society of America B: Optical Physics, vol. 20, no. 10, pp. 2052-2060, 2003.

[23] R. R. Adhami, D. J. Lanteigne, and D. A. Gregory, "Photopolymer hologram formation theory," Microwave and Optical Technology Letters, vol. 4, no. 3, pp. 106-109, 1991.

[24] V. L. Colvin, R. G. Larson, A. L. Harris, and M. L. Schilling, "Quantitative model of volume hologram formation in photopolymers," Journal of Applied Physics, vol. 81, no. 9, pp. 59135923, 1997.
[25] G. M. Karpov, V. V. Obukhovsky, T. N. Smirnova, and V. V. Lemeshko, "Spatial transfer of matter as a method of holographic recording in photoformers," Optics Communications, vol. 174, no. 5-6, pp. 391-404, 2000.

[26] S. Meng, H. Duran, J. Hu et al., "Influence of photopolymerization reaction kinetics on diffraction efficiency of H-PDLC undergoing photopatterning reaction in mixtures of acrylic monomer/nematic liquid crystals," Macromolecules, vol. 40, no. 9, pp. 3190-3197, 2007.

[27] S. Gallego, A. Márquez, M. Ortuño, S. Marini, and J. Francés, "High environmental compatibility photopolymers compared to PVA/AA based materials at zero spatial frequency limit," Optical Materials, vol. 33, no. 3, pp. 531-537, 2011.

[28] M. Ortuño, M. Riquelme, S. Gallego, A. Márquez, I. Pascual, and A. Beléndez, "Overmodulation control in the optimization of a H-PDLC device with ethyl eosin as dye," International Journal of Polymer Science, vol. 2013, Article ID 357963, 8 pages, 2013.

[29] Y. J. Liu and X. W. Sun, "Holographic polymer-dispersed liquid crystals: Materials, formation, and applications," Advances in OptoElectronics, vol. 2008, Article ID 684349, 52 pages, 2008.

[30] S. Gallego, M. Ortuño, C. Neipp et al., "Physical and effective optical thickness of holographic diffraction gratings recorded in photopolymers," Optics Express, vol. 13, no. 6, pp. 1939-1947, 2005.

[31] M. G. Moharam, E. B. Grann, D. A. Pommet, and T. K. Gaylor, "Formulation for stable and efficient implementation of the rigorous coupled-wave analysis of binary gratings," Journal of the Optical Society of America A: Optics and Image Science, and Vision, vol. 12, no. 5, pp. 1068-1076, 1995.

[32] C. Neipp, J. T. Sheridan, S. Gallego et al., "Effect of a depth attenuated refractive index profile in the angular responses of the efficiency of higher orders in volume gratings recorded in a PVA/acrylamide photopolymer," Optics Communications, vol. 233, no. 4-6, pp. 311-322, 2004.

[33] M. Ortuño, S. Gallego, A. Márquez, C. Neipp, I. Pascual, and A. Beléndez, "Biophotopol: a sustainable photopolymer for holographic data storage applications," Materials, vol. 5, pp. 772-783, 2012.

[34] Y. Liu, B. Zhang, Y. Jia, and K. Xu, "Improvement of the diffraction properties in holographic polymer dispersed liquid crystal Bragg gratings," Optics Communications, vol. 218, no. 1-3, pp. 27-32, 2003.

[35] H. Kogelnik, "Coupled wave theory for thick hologram gratings," Bell Systems Technology Journal, vol. 48, no. 9, pp. 29092947, 1969. 

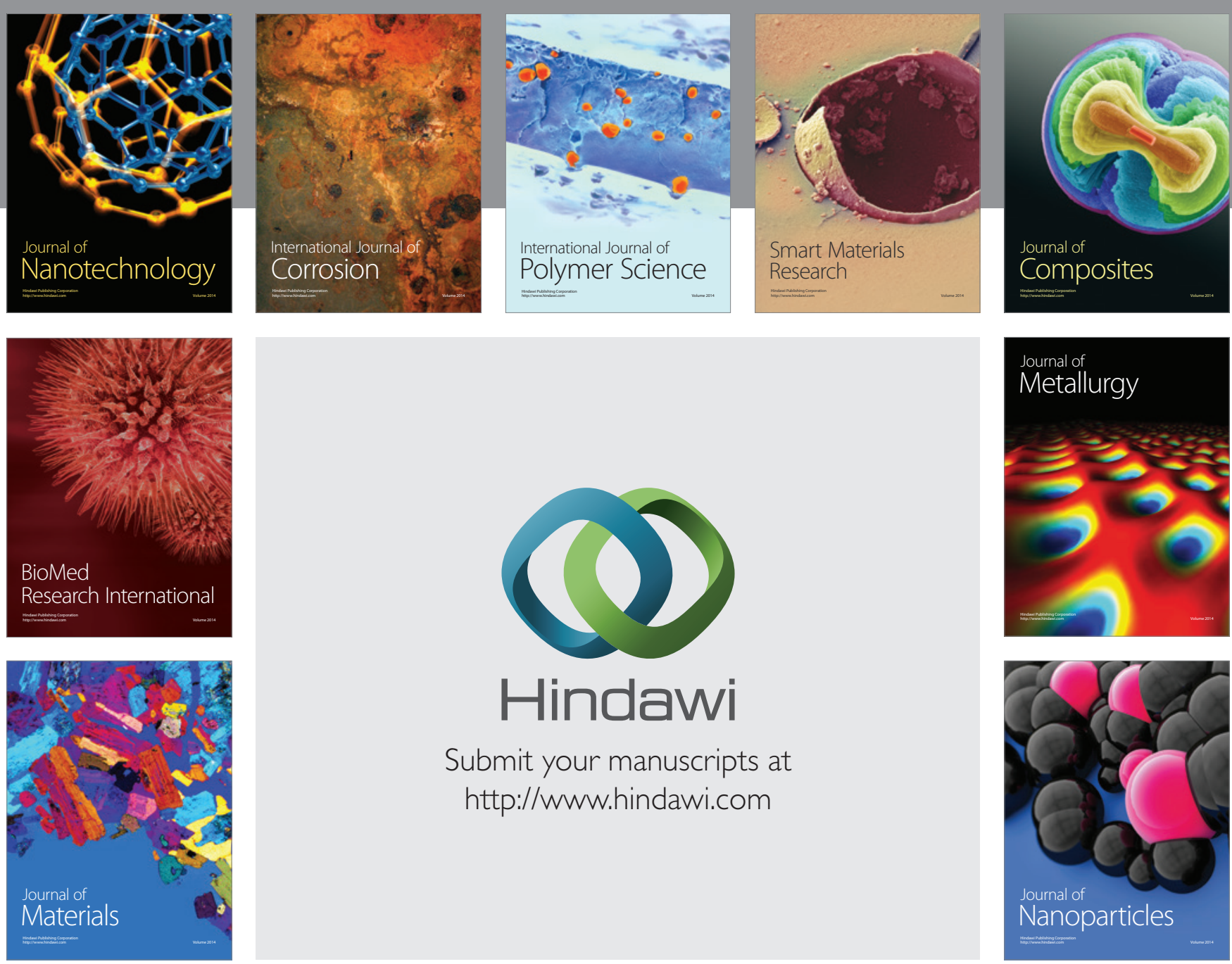

Submit your manuscripts at http://www.hindawi.com
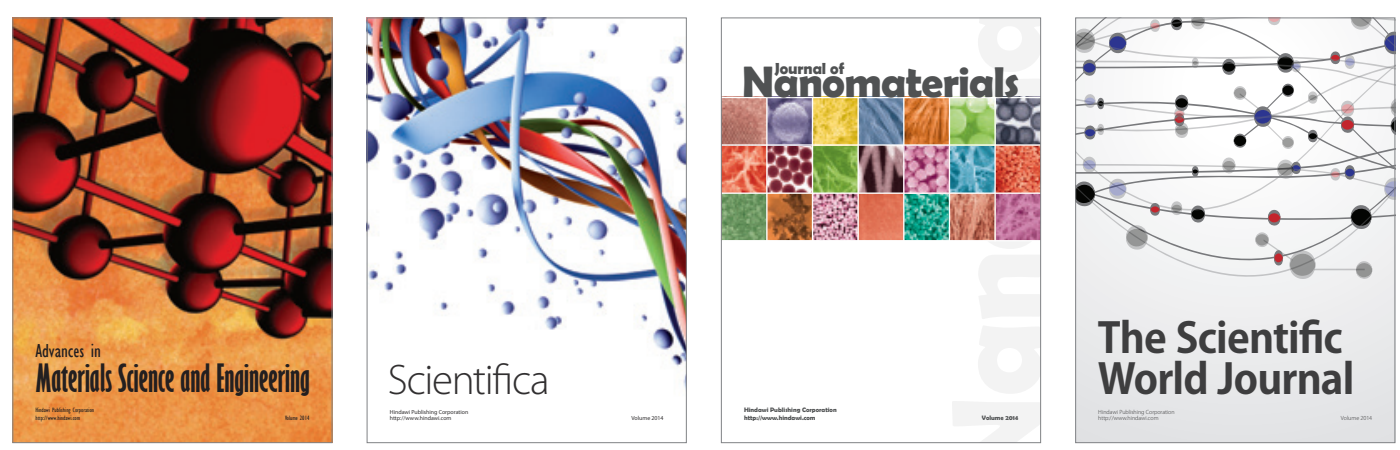

\section{The Scientific World Journal}
